\title{
High-repetition-rate Femtosecond-laser Inscription of Low-loss Thermally Stable Waveguides in Lithium Niobate
}

\author{
Teerawat Piromjitpong ${ }^{* a}$, Mykhaylo Dubov ${ }^{\mathrm{b}}$, and Sonia Boscolo ${ }^{\mathrm{a}}$ \\ ${ }^{a}$ Aston Institute of Photonic Technologies, School of Engineering and Applied Science, Aston University, Birmingham B4 7ET, UK; \\ ${ }^{b}$ Optoscribe Ltd., Livingston EH54 7EJ, UK; * piromjit@aston.ac.uk
}

\begin{abstract}
Optical-lattice-like WGs were fabricated in z-cut $\mathrm{LiNbO}_{3}$ by $\mathrm{HRR}$ pulse laser. Low propagation loss was observed in both orthogonal polarizations in the visible and near-IR spectrum. Single-mode guiding was maintained after high-temperature annealing.

OCIS codes: (130.0130) Integrated optics; (130.3730) Lithium niobate; (140.3390) Laser material processing
\end{abstract}

Owing to its robustness and unique flexibility, the direct femtosecond (fs) laser inscription method [1] is one of the most efficient techniques for three-dimensional volume micro-structuring of transparent dielectrics. The focus of this work is on the fabrication and optimization of depressed-cladding waveguides in a lithium niobate (LN) crystal. Our deployment of a high-repetition-rate (HRR) fs-laser enables relatively shorter processing times compared to its lowrepetition-rate (LRR) counterpart, which is especially advantageous to the manufacture of complex structures and large-scale commercial production.

The inscribing laser was a Ti:Sapphire oscillator (Femtolasers) that delivered an output beam at 790 -nm central wavelength and 11-MHz repetition-rate. The pulse duration was adjusted to $66 \mathrm{fs}$, corresponding to a spectral width of $25 \mathrm{~nm}$. A 100x microscope objective with a numerical aperture of 1.25 was used to focus the beam beneath the polished surface of 5\% magnesium-doped z-cut LN wafers. Fig. 1L shows an example waveguide cross-section where the array of circles represents the track positions. Each waveguide comprised 84 tracks precisely arranged in an optical-latticelike packing geometry with four hexagonal layers. The tracks were damage tracks, and were inscribed via a single scan along the LN crystalline X-axis. We deployed two new approaches to enhance the inscription results: 1) increase of the pulse energy with increasing focus depth inside the material to compensate for the corresponding decrease of refractiveindex (RI) modification, and 2) decrease of the laser energy for the modification tracks closer to the waveguide's core region to reduce scattering losses due to high-laser-energy driven non-uniformities. Hence, the pulse energy allocated to the machining of a track of vertical position $m$ and radial position $n$ can be described by the following equation:

$$
E_{m, n}=E_{0}+m \Delta E_{d}+\left(\frac{n}{3}\right)^{p}\left(\Delta E_{r}\right)
$$

where the energy-increase steps $\Delta E_{d}$ and $\Delta E_{r}$, the initial pulse energy $E_{0}$, and $p$ are given in Fig.1L for different waveguide groups (A, B, BX and C), classified according to their inscription conditions. Details can be found in [2].

The near-field intensity profiles captured by the InGaAs camera (Goodrich) and various fibre-coupled light sources with the wavelength range of $532-1550 \mathrm{~nm}$, showed that the fundamental guiding mode was the dominant mode excited in all waveguides being studied. Slightly tuning of the relative light coupling-stage positions only resulted into minor changes in mode shape and circularity, but did not cause excitation of higher-order modes. Although the A05 and B05 waveguides were fabricated with the same initial pulse energy $\left(E_{0}=48.2 \mathrm{~nJ}\right)$, the tracks on the upper and lower rows of B05 were apparently more consistent in size and strength of modification areas than those of A05 (Fig. 1M), thereby leading to higher and more symmetric light confinement. In fact, the measured propagation losses and mode-field
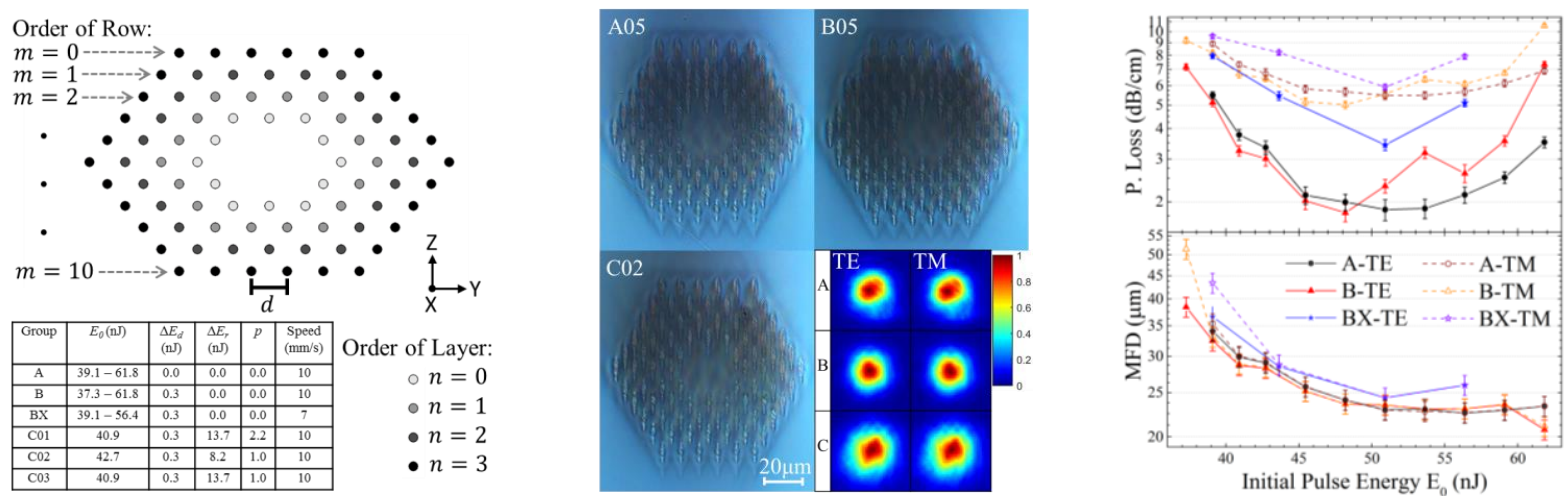

Fig. 1. (L) Cross-sectional layout of a waveguide, where the circles represent the track positions written in the inscription computer code. The table shows the inscription parameters for different groups of fabricated waveguides. (M) Differential-interference-contrast (DIC) images of the cross-sections of different waveguides, and corresponding near-field intensity distributions in the TE and TM polarizations measured at $1550 \mathrm{~nm}$. (R) Propagation losses and MFD versus initial pulse energy $E_{0}$ at $1550 \mathrm{~nm}$ before thermal annealing. 

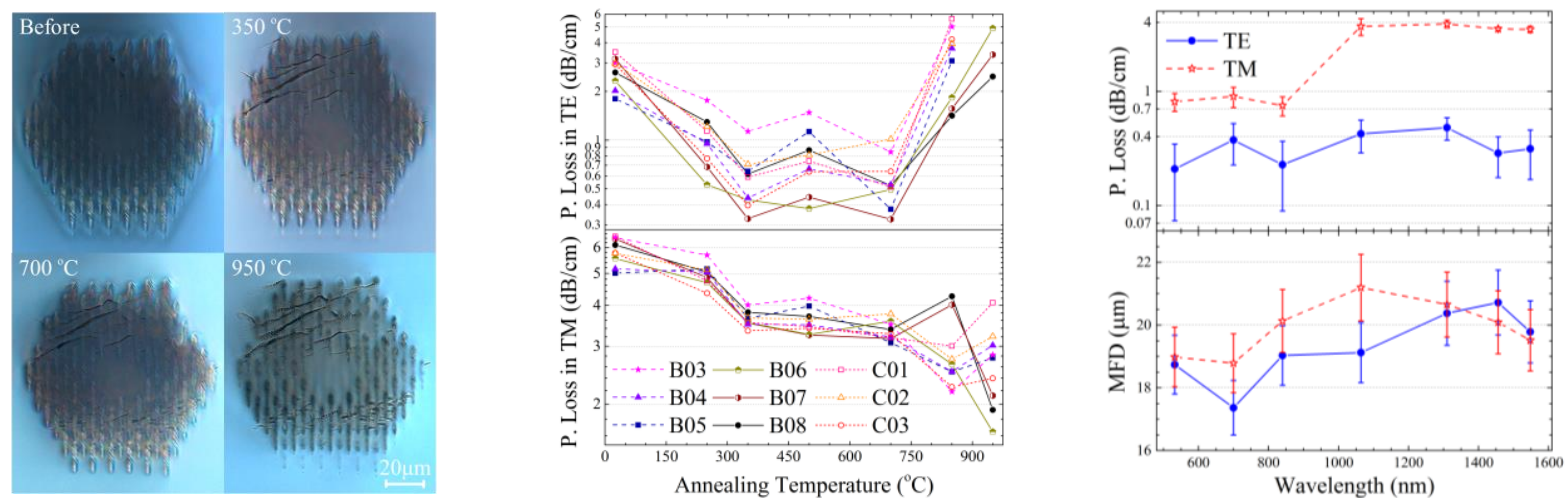

Fig. 2. (L) DIC images of the B06 waveguide cross-section before thermal annealing and after different thermal treatments. (M) Propagation losses versus annealing temperature at $1550 \mathrm{~nm}$ for different waveguides. (R) Wavelength-dependence of propagation losses and MFD for the B06 waveguide after the $2^{\text {nd }}$ thermal annealing step at $350{ }^{\circ} \mathrm{C}$.

diameter (MFD) of B05 $\left(\alpha_{\mathrm{TE}}=1.8 \pm 0.1 \mathrm{~dB} / \mathrm{cm}, \alpha_{\mathrm{TM}}=5.0 \pm 0.2 \mathrm{~dB} / \mathrm{cm}, \mathrm{MDF}=27.6 \pm 1.4 \mu \mathrm{m}\right)$ revealed enhanced beamguiding performance compared to A05 $\left(\alpha_{\mathrm{TE}}=2.0 \pm 0.2 \mathrm{~dB} / \mathrm{cm}, \alpha_{\mathrm{TM}}=5.6 \pm 0.2 \mathrm{~dB} / \mathrm{cm}, \mathrm{MDF}=28.2 \pm 1.4 \mu \mathrm{m}\right)$, which confirmed the benefits of our fabrication approach. The same trends were also observed for the waveguides inscribed with the lower pulse energy (Fig. 1R; $E_{0}=37.3-48.2 \mathrm{~nJ}$ ). In the high pulse energy regime $\left(E_{0}=50.9-61.8 \mathrm{~nJ}\right)$, groupA waveguides showed less attenuation, which we attribute to a smaller amount of high-energy-driven defects and weaker induced stress field. In comparison to groups $\mathrm{A}$ and $\mathrm{B}$, the waveguides in group $\mathrm{C}$ demonstrated significantly higher propagation losses and larger MDF, which can be explained by lower induced RI contrast and smaller size of the tracks in the inner layer. The inferior guiding performance in group BX is likely to be due to higher track roughness and stronger induced stress field that were typically observed for fabrication at lower translation speeds.

We performed heat treatments of the waveguides at different temperatures in an incremental order and with a duration time of 3 hours each. Fig. 2M shows the significant enhancement of the optical guiding performance in both TE and TM polarizations at 1550-nm wavelength afforded by thermal annealing, especially by the first two steps $\left(250^{\circ} \mathrm{C}\right.$ and $\left.350^{\circ} \mathrm{C}\right)$. The propagation losses decreased to a minimum after the sample was annealed at $350{ }^{\circ} \mathrm{C}$, and then slightly increased after the application of higher temperatures up to $700^{\circ} \mathrm{C}$. The lowest propagation losses of less than $0.4 \pm 0.1 \mathrm{~dB} / \mathrm{cm}$ and $3.5 \pm 0.2 \mathrm{~dB} / \mathrm{cm}$ in the TE and TM polarizations, respectively, were achieved from the B07 waveguide $\left(E_{0}=53.6 \mathrm{~nJ}\right)$. These losses were calculated from the measured insertion loss of $3.7 \mathrm{~dB}$, the mode-mismatch loss of approximately $1.5 \mathrm{~dB}$, and the double-Fresnel loss, which was estimated to be $1.4 \mathrm{~dB}$ for the air-LN interfaces. The MFD exhibited a similar behavior with varying annealing temperature, but it started to increase earlier, just after the second annealing step $\left(350^{\circ} \mathrm{C}\right)$. The improvement in guiding properties induced by thermal annealing can be explained by a stronger light confinement due to an increased overall RI contrast, and a weaker induced stress field as well as reduced transient defects. The light confinement in the TE polarization notably diminished after the annealing step of $850{ }^{\circ} \mathrm{C}$ and started to vanish after $950{ }^{\circ} \mathrm{C}$ in some low laser-energy-induced waveguides (B03-05 and C01-03). Interestingly, these waveguides continued to confine the beam in the TM polarization even after these high-temperature treatments. Fig. 2R shows the wavelength-dependence of the guiding characteristics for the B06 waveguide $\left(E_{0}=50.9\right.$ $\mathrm{nJ})$. While the propagation loss in the TE polarization remained approximately constant over the wavelength range being studied, the loss in the TM polarization changed to higher values around the boundary between the visible and near-infrared regions. The MDF in both TE and TM polarizations tended to increase with increasing wavelength.

In conclusion, we have demonstrated the HRR femtosecond-laser inscription of waveguides in z-cut LN that featured low propagation losses in both TE and TM polarization states and preserved nonlinearity. Single-mode guidance was observed across a wide portion of the spectrum from visible to near infrared (532 nm to $1550 \mathrm{~nm}$ ). Two new approaches based on varying the inscribing laser pulse energy in accordance with the position of the tracks were deployed in the fabrication process, which led to enhancement of the waveguide's confinement and intensity distribution symmetry. The heat treatment of the fabricated waveguides revealed an interesting feature of the damage tracks generated by a HRR fs-laser: the RI contrast is enhanced by annealing and remains stable under high temperatures up to $700{ }^{\circ} \mathrm{C}$, opposed to the track degradation that typically occurs in the case of LRR-laser inscription. The low attenuation and high thermal stability of our waveguides suggest promising applications in telecommunications, nonlinear integrated optics, and harsh environment sensors.

\section{References}

[1] S. Gross et al., "Ultrafast-laser-inscribed 3D integrated photonics: challenges and emerging applications," Nanophotonics 4, 332-352 (2015).

[2] T. Piromjitpong et al., "High-repetition-rate femtosecond-laser micromachining of low-loss optical-lattice-like waveguides in lithium niobate," in Nonlinear Optics and Its Applications (2018), Vol. 10684 of SPIE Proceedings Series, pp. 106840D. 